\title{
Cost Efficient Localized Geographical Forwarding Strategies for Wireless Sensor Networks
}

\author{
Michele Rossi ${ }^{1}$ and Michele Zorzi ${ }^{2}$ \\ 1 Department of Engineering, University of Ferrara, \\ via Saragat 1, \\ 44100 Ferrara, Italy \\ mrossieing.unife.it \\ 2 Department of Information Engineering, University of Padova \\ via Gradenigo 6B, \\ 35131 Padova, Italy \\ zorzi@ing.unife.it
}

\begin{abstract}
In this paper we investigate the relationship between local next hop selection strategies and their efficiency in terms of both link related metrics, such as the mean packet delivery fraction, and network related metrics, such as the energy status of the node elected as relay. In standard geographical forwarding algorithms the relay selection is usually carried out by means of advancements toward the destination. However, channel attenuation phenomena often make pure geographical strategies ineffective as the quality of a transmission link is not necessarily deterministically related to the node coordinates. In order to achieve effective and cost efficient routing solutions, it is therefore crucial to couple advancements toward the destination with link quality aspects as well as network related metrics (e.g., node energies). This study is a preliminary step toward the design of local relay selection rules which jointly account for these aspects and whose aim is to cut the desired trade-off between delay and cost efficiency.
\end{abstract}

Keywords: Wireless sensor networks, routing, MAC techniques, cross-layer design, performance evaluation.

\section{Introduction}

Geographical routing is a key concept which is very often considered for data forwarding in multi-hop wireless sensor networks (WSNs) [1] and Ad Hoc networks [2]. Many routing solutions, in fact, exploit the concept of maximum advancements toward the destination [3-6] to effectively route packets in a best effort (greedy) manner. However, recent empirical measurements [7-9] have proved that the unit disk connectivity model [10], on which these solutions are based, often fails in real settings. In particular, channel attenuation phenomena such as e.g. multi-path fading [11], invalidate the unit disk connectivity assumption, thereby heavily affecting the good results obtained so far for pure geographical routing schemes. In this work, we remove the unit disk model assumption, by going in the direction of recent research [12] and studying the impact of a more accurate connectivity model on the metric to be used to implement geographical forwarding. The impact of fading on geographical random forwarding has been 
studied in [13]. Our aim here is to account for real fading statistics and derive exact formulae to properly weigh the nodes geographical advancements toward the destination in a faded channel. Subsequently, we use such statistics to drive the relay node (next hop) election by accounting for the "expected advancements", that we define here as the product of the actual geographical advancements and the related packet success rates [14]. In addition, we also account for the so called network costs, that we use in the present contribution to model node specific quantities such as residual energies and/or congestion states. In our framework, link specific costs are accounted for by the above mentioned expected advancements, whereas node specific costs, such as residual energies, are taken into account by the network costs.

In the present paper, we propose a novel relay contention scheme, where all nodes with a good expected advancement metric are first collected; our analytically derived curves on the optimal expected advancements are used to this end. Subsequently, these nodes are involved in the relay election phase, which is performed by means of a probabilistic back-off scheme and whose aim is to promote the node with the lowest network cost. The original aspects of our contribution consist of both the greater accuracy of our analytical derivations with respect to previous results [12] as well as of the novelty of the proposed channel contention procedure for the election of the relay node. Our derivations for the optimal advancement metric are in line with [14]; however, we do not consider the interference due to out of range nodes, and we also derive the statistics with a different perspective, i.e., conditioned on the actual advancement of a given node in the forwarding region. In fact, sensor networks are expected to deal with low traffic communications and therefore in these scenarios this type of interference is less important. We instead still focus on channel fading and its consequences to the achievable advancements within a given local relay election phase. Our work is also very much in line with [15], where the authors also stress the importance of keeping the packet error rate into account in geographical forwarding. The main differences of our approach with respect to [15] consist in the novel MAC contention procedure that we propose in Section 4 , as well as the new probabilistic filtering procedure that we propose to pick the nodes with the highest expected advancement within range. Furthermore, we remark that in our study we explicitly consider the correlation among nodes costs by showing the impact of this metric on the relay election procedure. To the best of our knowledge, the cost correlation has never been considered before in the design of contention algorithms for WSNs. However, this is a crucial metric that has to be taken into account when the objective is to elect "good" relay nodes, where the node goodness might be related to residual energies, congestion levels as well as data aggregation aspects. In certain settings, in fact, in order to optimally exploit the network resources it might be beneficial to elect a next hop which has data to aggregate, in spite of other requirements.

The remainder of the paper is organized as follows: in Section 2 we present the system model that will be subsequently considered to carry out our analytical derivations. In Section 3 we analytically derive the relationship between geographical and expected advancements. In Section 4 we propose a novel channel contention phase for the local election of relay nodes while in Section 5 we discuss the impact of the cost correlation on the relay selection procedure. In Section 6 we report some results by comparing our scheme to previously proposed solutions that exploit the pure geographical advancement metric and finally, in Section 7, we report the conclusions of our work. 


\section{System Model}

Throughout the paper we will make the following assumptions:

1. Topology: We model the network as a weighted graph $\mathcal{G}=(\mathcal{N}, \mathcal{L})$, consisting of a set $\mathcal{N}$ of nodes and a set $\mathcal{L}$ of arcs, where we refer to $l_{i j} \in \mathcal{L}, i, j \in \mathcal{N}$, as the link connecting node $i$ with node $j$. We consider bi-directional links and we say that a link between the two nodes $i$ and $j$ exists with probability $P_{s}\left(d_{i j}\right)$, where $P_{s}(\cdot)$ corresponds to the probability of successfully transmitting a data packet from node $i$ to node $j$ and is calculated as a function of the distance $d_{i j}$ separating the two nodes. The characterization of $P_{s}(\cdot)$ is detailed below. For the topology, we assume that nodes are distributed according to a planar Poisson process with intensity $\rho$ users per unit area [16]. That is, the probability of having $n \in \mathbb{N}$ devices within an area $\mathcal{A} \in \mathcal{R}^{+}$is given by $\mathcal{P}(n, \rho, \mathcal{A})=\left((\rho \mathcal{A})^{n} / n !\right) \exp (-\rho \mathcal{A})$.

2. Channel model: for the channel model, we consider both path loss attenuation and fast fading, which is modeled here by means of the Rayleigh fading statistics [11]. For the sake of illustration, let us refer to the communication between node F (forwarder) and node $N$ (next hop) in Fig. 1. If the distance between the two nodes is $r$, then the probability that node $\mathrm{N}$ will receive the packet transmitted by $\mathrm{F}$ is calculated as:

$$
P_{s}(r)=\operatorname{Prob}\left\{\alpha r^{-\eta} \geq b\right\}
$$

where $\eta$ is the path loss propagation exponent, usually within the range $\eta \in[2,4]$, $\alpha$ is the fading value for a given packet transmission ${ }^{3}$ and $b$ is a technology dependent threshold used to model the probability that the received signal envelope is successfully decoded. We further define $R$ as the transmission range value for which $P_{s}(R)=\zeta$, where $\zeta$ is a small probability value. We refer to $R$ as the maximum transmission range, by probabilistically modeling the fact that for communication distances longer than $R$ the transmitted data is likely to be corrupted. In practice, we use $R$ to model the minimum acceptable level of QoS (quality of service). The following analytical framework will rely on this assumption, i.e., the derived results will be conditioned on assuming $R$ as the maximum transmission range: nodes placed at longer distances are not considered as possible relay nodes.

3. Radio activities: We allow nodes to periodically switch between awake and sleeping modes, where they can switch off the radio activity for energy saving purposes. If we express the duty cycle $t_{o n}$ as the fraction of time in which nodes are in the active state, then at every data forwarding stage the only nodes that can be considered for data routing are the ones actually awake within the forwarding range $R$. By considering independent on/off radio cycles at every node, this fact is modeled through an equivalent Poisson process of density $\rho_{\text {on }}=\rho t_{\text {on }}$, which gives the average number of awaken nodes per unit area at a given instant.

4. Nodes advancements: Consider the node advancement diagram illustrated in Fig. 1, where we represent a snapshot of the routing process for a given data packet. In particular, node $\mathrm{F}$ has to select a next hop $\mathrm{N}$ to act as a relay for the current packet. In our setting, data forwarding is achieved on the fly, by only exploiting local knowledge about network topology and nodes costs. For what concerns the topology

\footnotetext{
${ }^{3}$ We reasonably assume that the attenuation due to fading remains constant during a packet transmission, but is uncorrelated among subsequent transmission events (block fading model).
} 
aspect, F should select the node leading to the maximum expected advancement toward the sink (destination). For illustration purpose, suppose there are $M$ neighbors lying in the forwarding area (half circle with radius $R$, toward the sink) and that their distances from $\mathrm{F}$ are $\left(r_{1}, r_{2}, \ldots, r_{M}\right)$. Let $\left(z_{1}, z_{2}, \ldots, z_{M}\right)$ be the vector of projected distances toward the sink. A locally optimal geographical forwarding is therefore achieved by selecting node $i^{\star}$ such that:

$$
i^{\star}=\operatorname{argmax}_{j \in\{1,2, \ldots, M\}}\left\{z_{j} P_{s}\left(r_{j}\right)\right\}
$$

In fact, in our setting the correct way of dealing with geographical advancements is to account for expected advancements, which are achieved as $z_{j} P_{s}\left(r_{j}\right)$. Observe that this leads to a substantially different analysis from the unit disk [10] propagation model, where transmissions to the nodes placed within the transmission range are always successful and the only cause of error is packet collision. In the following derivations, we refer to the forwarding area, the half circle with radius $R$ toward the sink in Fig. 1, as $\mathcal{F}$. It is important to stress that expected advancements will only be used in the initial phase of the protocol that we propose in the present paper and with the aim of picking in a distributed fashion the most suitable relay candidate. Furthermore, in a subsequent contention phase, these nodes will further contend for the relay election by means of a properly designed backoff algorithm, where the choice of the relay will be driven by the so called network costs, i.e., by jointly accounting for nodes geographical advancements and nodes residual energies, as addressed in the following point. In practice, the choice of the relay is a two-step process where we first discriminate among nodes with a good expected advancement metric and we subsequently refine our choice of the relay node by also accounting for network related aspects such as residual energies. This second phase is driven by the node costs presented below.

5. Node cost: These are the costs considered in the second contention phase of the joint MAC/routing protocol that will be presented in Section 4. We define nodes costs so as to encode several aspects of the communication. First of all, they must reflect geographical advancements, as our objective is to route packets toward the destination using node coordinates. (Note that in this case we use again the advancement, which is then considered in both contention phases.) However, it shall be observed that advancements are not the only quantities to be accounted for. In fact, one may also think of optimizing other factors such as residual energies and congestion levels. These metrics are indeed important to discriminate among nodes with the same advancement metric and therefore implement a "network" efficient choice of the relay node. This "network consciousness" refers to the fact of jointly accounting for possibly heterogeneous factors so as to pick the nodes with good advancements (current communication perspective) but also with other desirable properties (e.g. residual energies) and this is done with the aim of optimizing the network utilization (network perspective). In order to implement the above requirements, here we associate a normalized finite cost $c_{i}$ to every node $i \in \mathcal{N}$, where without loss of generality $c_{i} \in[0,1]$. Observe that these costs are independent of the link quality and are node specific. For these costs, we introduce a flexible definition which accounts for both advancement and residual energy aspects. Accordingly and without loss of generality, in this contribution we will express the costs for the generic node 


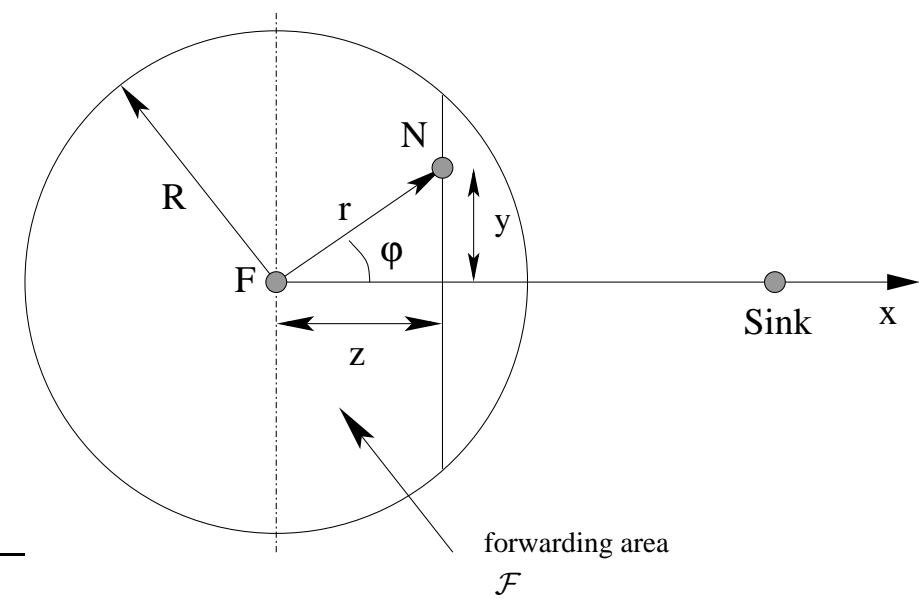

Fig. 1. Diagram for the considered nodes geographical advancement model.

$i \in \mathcal{N}$ as follows:

$$
c_{i}=\xi\left(1-E_{i} / E_{\text {init }}\right)+(1-\xi)\left(1-z_{i} / R\right)
$$

where $\xi \in[0,1]$ is a factor used to weigh the relative importance of the two terms, $E_{i}$ is the residual energy reserve at node $i, E_{\text {init }}$ is the initial energy reserve and $z_{i}$ is the advancement toward the destination associated with node $i$. It shall be observed that in the costs one might also encode further factors such as congestion levels; we refer here to energy levels only as an example. Further investigation in this direction is the object of our current and future work.

\section{Characterization of Optimal Advancements}

Let us refer to Fig. 1. If $r$ is the distance between the sender (F) and a given receiver in the forwarding area $\mathcal{F}$, its pdf ${ }^{4}$ is derived as $f(r)=2 r / R^{2}$. Moreover, if $Z$ is the r.v. governing the projected advancement toward the sink, its pdf conditioned on $r$ is given by [17]:

$$
f_{Z}(z \mid r)= \begin{cases}0 & r<z \\ \frac{2}{\pi r \sqrt{1-z^{2} / r^{2}}} & 0 \leq z \leq r \leq R\end{cases}
$$

Now, we further define $\Xi$ as the r.v. of the actual advancement $\xi_{i}=z_{i} P_{s}\left(r_{i}\right)$ for the generic node $i$ in the forwarding region $\mathcal{F}$. It follows that the cdf associated with $\Xi$, $\operatorname{Prob}\{\Xi \leq x\}$, is given by:

$$
\begin{aligned}
F_{\Xi}(x) & =\int_{0}^{R} f(r) \int_{0}^{x / P_{s}(r)} f_{Z}(z \mid r) \mathrm{d} z \mathrm{~d} r= \\
& =\frac{4}{\pi R^{2}} \int_{0}^{R} r \arcsin \left\{\frac{\min \left[r, x / P_{s}(r)\right]}{r}\right\} \mathrm{d} r
\end{aligned}
$$

\footnotetext{
${ }^{4}$ Conditioned on the maximum range $R$ defined as above.
} 


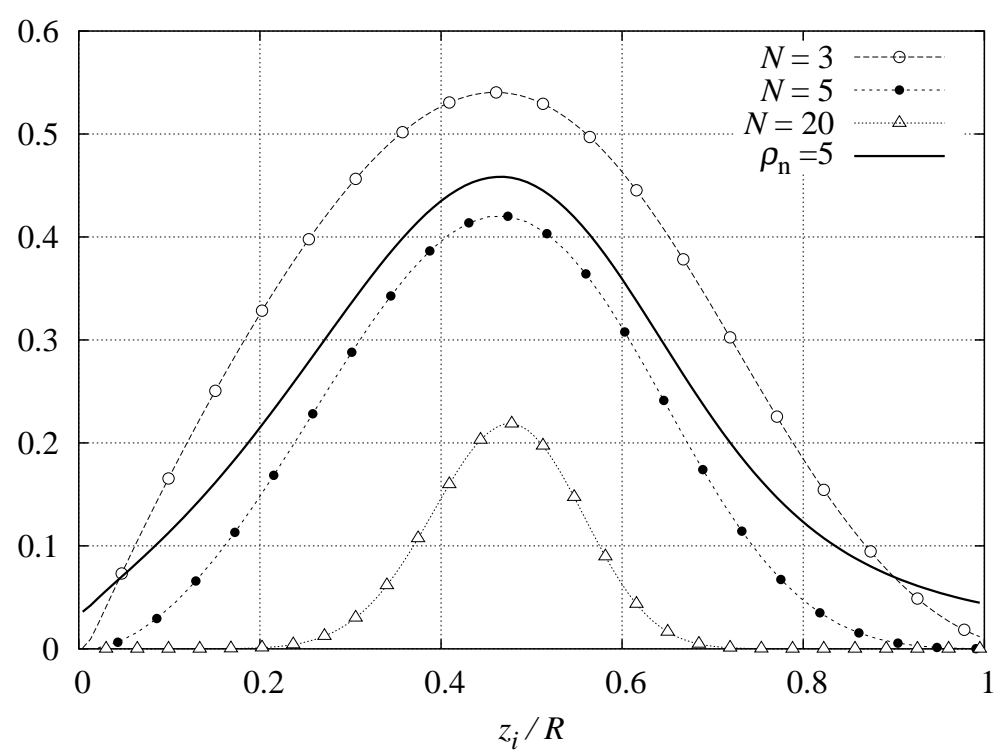

Fig. 2. $\Gamma\left(z_{i}, N\right)$ by varying $N \in\{3,5,20\}$ and $\Gamma\left(z_{i}\right)$ for $\rho_{n}=5 . \zeta=0.01, \eta=4$.

Moreover, referring to $y$ as the distance between node $\mathrm{N}$ and the line connecting $\mathrm{F}$ to the sink (Fig. 1) and applying the uniformity property of the Poisson process we have that the pdf $f_{Y}(y \mid z)$ conditioned on a given advancement $z$ is:

$$
f_{Y}(y \mid z)= \begin{cases}\frac{1}{2 \sqrt{R^{2}-z^{2}}} & y_{\min }(z) \leq y \leq y_{\max }(z) \\ 0 & \text { elsewhere }\end{cases}
$$

where $y_{\min }(z)=-\sqrt{R^{2}-z^{2}}$ and $y_{\max }(z)=\sqrt{R^{2}-z^{2}}$. Now, if we consider a number $N$ of users in $\mathcal{F}$, the probability $\Gamma\left(z_{i}, N\right)$ that a given device $i \in\{1,2, \ldots, N\}$ with given geographical advancement $z_{i}$ is the one leading to the highest expected advancement $\xi_{i^{\star}}$ (see Eq. (2)) is obtained as follows:

$$
\Gamma\left(z_{i}, N\right)= \begin{cases}1 & N=1 \\ \int_{y_{\min }\left(z_{i}\right)}^{y_{\max }\left(z_{i}\right)} f_{Z}\left(y \mid z_{i}\right)\left[F_{\Xi}\left(z_{i} P_{s}\left(\sqrt{z_{i}^{2}+y^{2}}\right)\right)\right]^{N-1} \mathrm{~d} y & N>1\end{cases}
$$

Observe that the above probability is conditioned on $z_{i}$ and $N$. Moreover, the awake nodes in $\mathcal{F}$ can be modeled through a Poisson distribution with intensity $\rho_{\text {on }}$. Hence, we can use $\mathcal{P}\left(n, \rho_{\text {on }}, \pi R^{2} / 2\right)$ to average $\Gamma\left(z_{i}, N\right)$ over the number of awake nodes $N$ in $\mathcal{F}{ }^{5}$ Finally, we obtain $\Gamma\left(z_{i}\right)=\mathbb{E}_{N}\left[\Gamma\left(z_{i}, N\right)\right]$ which corresponds to the expected probability for a given node with advancement $z_{i}$ to be the "best" node in $\mathcal{F}$ when the active nodes in $\mathcal{F}$ are Poisson distributed with density $\rho_{\text {on }}$.

\footnotetext{
${ }^{5}$ The average has to be carried out for $N \geq 1$, as at least node $i$ must exist in $\mathcal{F}$.
} 


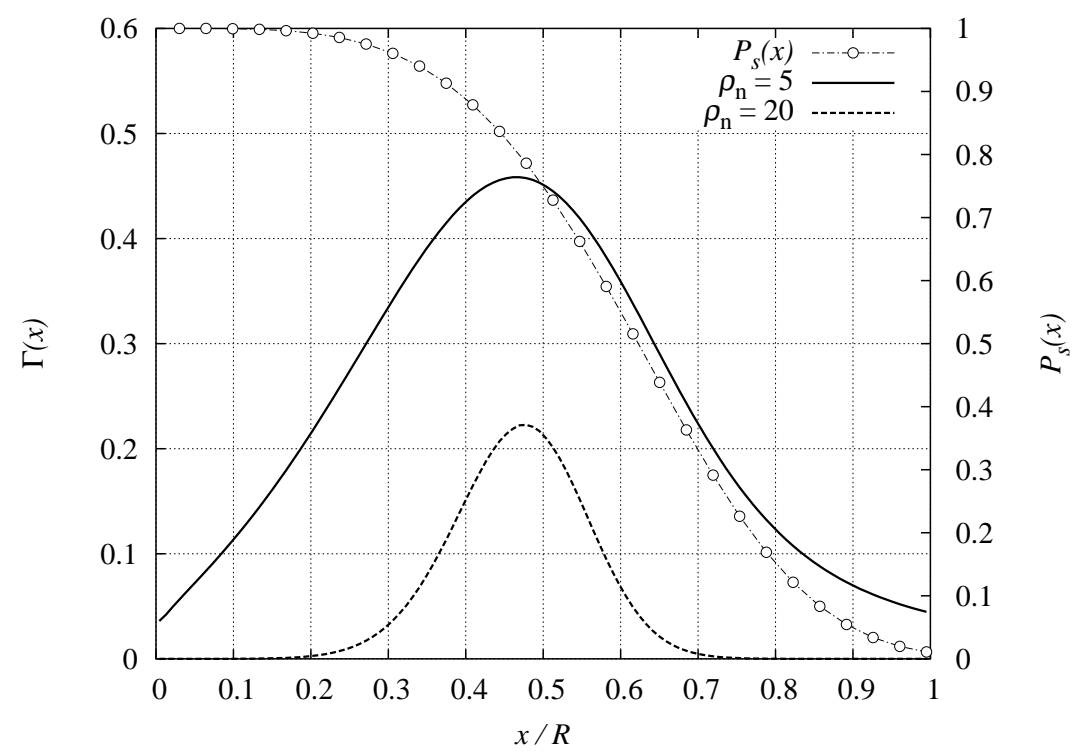

Fig. 3. $\Gamma\left(z_{i}\right)$ for $\rho_{n} \in\{5,20\}$. $P_{s}(r)$ is plotted for comparison. $\zeta=0.01, \eta=4$.

For illustration, in Fig. 2 we report $\Gamma\left(z_{i}, N\right)$ for different values of $N$, where we normalize the advancement $z_{i}$ to $R$. In the figure, we refer to the normalized density $\rho_{n}$ which is defined as the average number of awake nodes in $\mathcal{F}$, i.e., $\rho_{n}=\rho_{\text {on }}\left(\pi R^{2} / 2\right)$. As expected, with a fading channel the nodes close to the limit of the coverage range $R$ are not good candidates to be selected as relays for the packet transmission, as they will likely lead to small success probabilities ( $P_{s}(r)$ decreases as $r \rightarrow R$, see Fig. 3). On the other hand, if we pick a node $i$ with a small advancement $z_{i}$, we have that $P_{s}(r)$ is close to one but again the node is not a good relay candidate as $\xi_{i}\left(z_{i} P_{s}(r)\right) \rightarrow 0$ as $z_{i} \rightarrow 0$. Instead, for intermediate values of $z_{i}$, we have a so called transitional region [8] where nodes lead to good expected advancements toward the sink. This is indeed the most reasonable region to consider for the selection of relay nodes in geographical routing. In the following sections, we will discuss a possible way to exploit such a probability curve to implement effective relay selection schemes. In Fig. 3, we report both the success probability curve $P_{s}(x)$ and $\Gamma(x)$ as a function of the normalized distance $x / R$. Clearly, $P_{s}(x)$ drops as $x \rightarrow R$ and this is the reason for which the often considered $[6,18]$ maximum advancement within radius metrics does not represent the optimal relay node selection criterion when fading is taken into account. It shall be observed that our derivation is an extension of previous results. In fact, differently from the deterministic and one-dimensional topology considered in [12] we carry out the analytical calculation for a two dimensional case with a Poisson planar node distribution. This has the important advantage that the obtained probability curves, even if qualitatively in agreement with the results in [12], are more accurate as they reflect the true two-dimensional stochastic nature of a real forwarding environment and can therefore be directly used within practical forwarding schemes. 


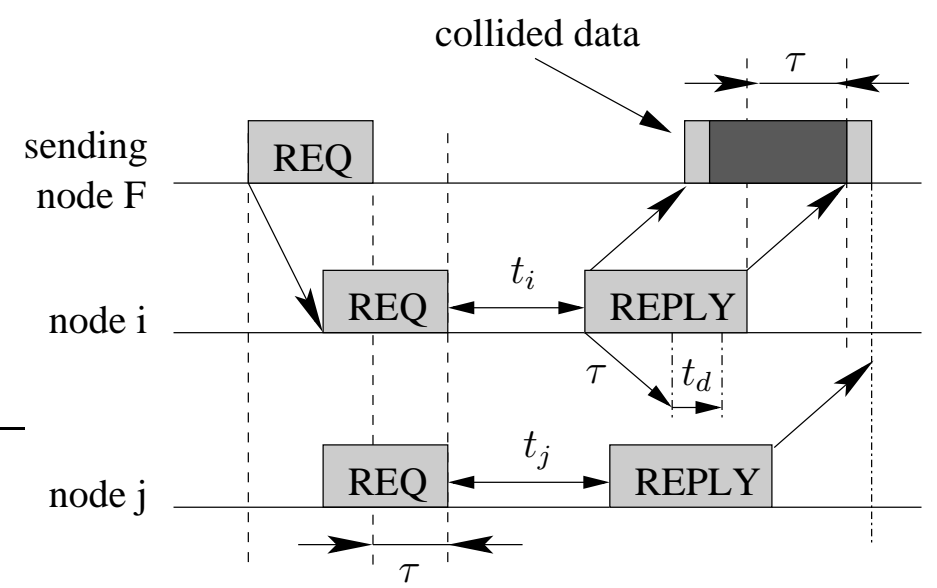

Fig. 4. Packet collision example in the relay node election phase.

\section{Proposal for a Coupled MAC/Routing Geographical Scheme}

In this section, we present an integrated MAC/routing solution that exploits the probability curves derived above to implement an efficient relay node election procedure. We assume that the sending device has an estimate for the density of awake nodes in its coverage area, $\rho_{\text {on }}$. Moreover, we assume that every node knows its own geographical position as well as the geographical coordinates of the sink. The problem to be solved is to carry out the selection of the relay node by jointly meeting the following requirements: 1) the relay node should have a good expected advancement metric, according to what discussed in the previous section, 2) the selected node should also have a good network cost metric, 3 ) the relay selection should be implemented such as to limit, as much as possible, the number of collisions ${ }^{6}$ associated with the relay election phase. In order to meet the first requirement, we advocate to use the function $\Gamma(\cdot)$ for implementing a probabilistic filtering of the number of nodes that will participate in the relay node selection phase. In particular, each node $j \in \mathcal{F}$ uses an estimate of the network density $\rho_{n}$ to properly select a $\Gamma(\cdot)$ curve and subsequently calculates the probability of being a good candidate to act as the relay for the current packet transmission; this probability is derived as $\Gamma\left(z_{j}\right)$. Hence, the awake nodes in $\mathcal{F}$ decide to participate in the following contention phase according to the probability $\Gamma\left(z_{j}\right)$; we refer to the set of these nodes as $\mathcal{S}$. Hence, we use such a probabilistic filtering to exclude from the channel contention those nodes that will likely lead to poor expected advancements. After this, we proceed with the actual election of the relay node. Within this second phase, each node in $\mathcal{S}$ calculates its own network cost, i.e., a mixture of residual energy and advancement as expressed by Eq. (3), and exploits this cost to derive the back-off value to be used in its subsequent access to the channel. In particular, we consider that a node $j \in \mathcal{S}$ transmits a message back to the sending node ( $\mathrm{F}$ in Fig. 1) with a time delay $t_{j}$

\footnotetext{
${ }^{6}$ Reducing the number of collisions corresponds to reducing the delay as well as the energy wastage in the contention procedure.
} 
which is computed as follows:

$$
t_{j}=c_{j} T_{1}+r_{j} T_{2}
$$

where $c_{j} \in[0,1]$ is the node cost as defined by Eq. (3), whereas $r_{j}$ is a random number in $\mathcal{U}[0,1]$, where with $\mathcal{U}[a, b]$ we indicate the uniform distribution in the interval $[a, b], a<b$. The parameters $T_{1}$ and $T_{2}$ can be set to adjust the performance of the channel contention by cutting the desired tradeoff between collision probability (duration of the contention) and quality of the solution found (cost of the node elected as relay). The setting of these parameters as well as their dependence on the cost statistics are addressed in greater detail in the following Section 5.

As a first step for the study of the contention scheme, we further consider the following two assumptions: 1) first of all, we do not account for the capture effect, i.e., we declare a collision whenever the reception of different packets overlaps at the receiver; 2) the second assumption relates to the carrier sensing, for which we assume that a node $i$ in the forwarding region $\mathcal{F}$ can always sense the ongoing transmission of another node $j$ in $\mathcal{F}$. Observe that this assumption is reasonable as the sensing range is in general higher than the transmission range. (If needed, the proposed protocol could be slightly modified to accommodate the uncommon situation in which assumption 2 is not verified.) Note that the effect of these two assumptions are a performance decrease (for point 1 ) and a performance increase (for point 2). Accurate evaluations by simulation have shown that the net effect is limited. A more detailed study is left for future research.

To track packet collisions, we refer to the channel propagation delay and to the minimum time required by the radio circuitry to detect an ongoing transmission as $\tau$ and $t_{d}$, respectively. Moreover, we express $t_{d}=n_{d} / B_{r}$, where $n_{d}$ is the number of subsequent bits to be received in order to detect an ongoing transmission, whereas $B_{r}$ is the communication bit-rate. If a collision occurs, i.e., the replies of two or more nodes in $\mathcal{S}$ partially overlap (see Fig. 4), then the collision is detected by the sending node that re-triggers a new contention round. In the new round, the sender also properly modifies $T_{1}$ and $T_{2}$ to decrease the collision probability, as will be discussed below. On the other hand, if node $i$ is the one selecting the smallest backoff $t_{i}$ and every other awake device in the forwarding area picks a back-off time $t_{j}$ such that $t_{j} \geq t_{i}+\tau+t_{d}$, $\forall j \in \mathcal{S}, j \neq i$, then the packet sent by node $i$ is received by the sending node with a probability $P_{s}(d)$, where $d$ is the distance between the sender and node $i$, and all the scheduled transmissions from any other node $j \in \mathcal{S}, j \neq i$ are canceled. ${ }^{7}$ The above procedure is repeated until a relay node is elected. After this, the sender forwards the current data packet to the selected relay. As an example, in Fig. 4 we plot the diagram for a collision event where the set $\mathcal{S}$ is composed by the two nodes $i$ and $j$. First of all, the sending node $\mathrm{F}$ starts the channel contention by sending a REQ message. This REQ triggers every node in $\mathcal{S}$ which independently computes its back-off time as explained above. Then, as the back-off expires, each node in $\mathcal{S}$ sends a REPLY back to F. In the figure, $t_{j}-t_{i}<\tau+t_{d}$ and therefore node $j$ does not have a sufficient time to detect the ongoing communication; its transmission after $t_{j}$ seconds from the reception of the REQ will therefore result in a collision at the sending node.

\footnotetext{
${ }^{7}$ Here, we exploit assumption 2, as every other node in $\mathcal{F}$ is able to sense an ongoing transmis-
} sion. 


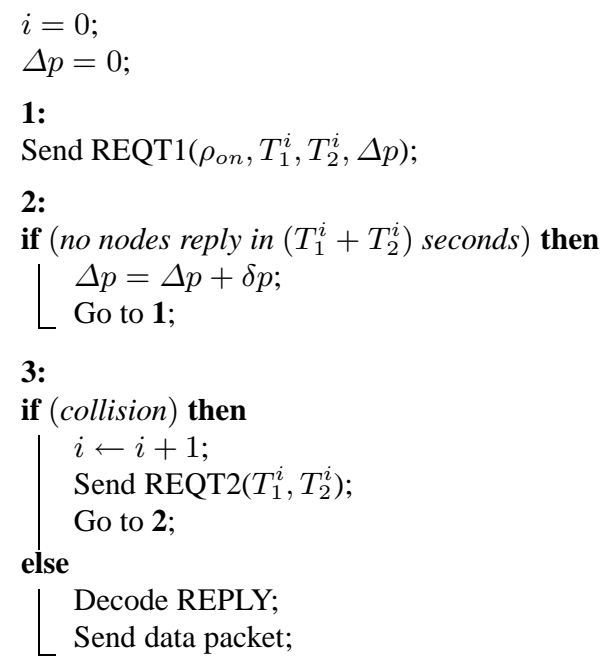

Algorithm 1: Algorithm executed by the sending node.

In Algorithms 1,2 and 3, we detail the relay selection procedure discussed above. Algorithm 1 describes the procedure executed by the sending node (F in Fig. 1). Node F starts the contention procedure by sending a REQ message of type 1 (REQT1), inclusive of the estimated node density $\rho_{n}$, of the two parameters $T_{1}$ and $T_{2}$ and of a constant $\Delta p$ whose meaning will be soon clarified. Each node in $\mathcal{S}$, after receiving a REQT1 packet (Algorithm 2) selects a $\Gamma(\cdot)$ curve depending on the value of $\rho_{n}$ contained in the request and decides to participate to the following contention phase with probability $\Gamma\left(z_{i}\right)+\Delta p$, where $z_{i}$ is the nodes own advancement. If all nodes in $\mathcal{F}$ decide not to participate in the subsequent channel contention, then $F$ will receive no REPLY. This situation should be unlikely as it means that all nodes lie either in a region very close to the forwarder (node $\mathrm{F}$ ) or close to the maximum transmission range $R$. In either case, in fact, the expected advancements $\xi=z P_{s}(r)$ are small and therefore lead to small access probabilities that, in turn, may cause such an "empty" transmission round. If an empty transmission round is detected, i.e., no REPLYs are received within a time interval of $T_{1}^{0}+T_{2}^{0}$ seconds, node $\mathrm{F}$ re-sends a further REQT1 message by inflating $\Delta p$ by the fixed quantity $\delta p \in[0,1]$. After this, every node in $\mathcal{F}$, upon receiving this second request, adds $\Delta p$ to $\Gamma\left(z_{i}\right)$, thereby increasing its probability of participating to the contention. This, on the long run, will force every node in the forwarding area to take part in the channel contention. We observe that $\Gamma(\cdot)$ is used here to shape the participation probabilities as a function of the expected advancements toward the sink. In such a way, we probabilistically advantage those nodes in the transitional region, by extending the possibility to take part in the channel access to less desirable nodes only if needed, i.e., if no candidates are found with a good expected advancement. After having decided to take part in the contention, a node $j \in \mathcal{S}$ initializes a back-off timer to $t_{j}$ according to Eq. (7) (see Algorithm 2) and, if no ongoing transmission from any other node is detected, transmits a REPLY back to F as its back-off expires. In the case of collision (Algorithm 1), F re-resends a REQ message of type 2 (REQT2), where it 


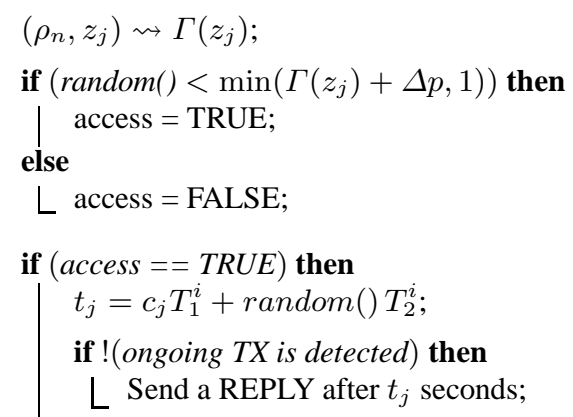

Algorithm 2: On receiving a $\operatorname{REQT1}\left(\rho_{n}, T_{1}^{i}, T_{2}^{i}, \Delta p\right)$ message at node $j \in \mathcal{S}$. random () generates a random number in $\mathcal{U}[0,1]$.

if $($ access $==$ TRUE) then

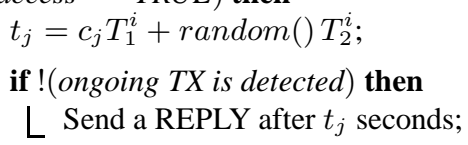

Algorithm 3: On receiving a REQT2 $\left(T_{1}^{i}, T_{2}^{i}\right)$ message at node $j \in \mathcal{S}$.

specifies new values for $T_{1}$ and $T_{2}$. As will be clarified by the results discussed in the following section, the adaptation of these two parameters is necessary to decrease the collision probability when nodes costs are correlated.

\section{Some Considerations on the Impact of the Statistical Properties of the Node Costs}

As the aim of this section is to understand the impact of the above introduced parameters $T_{1}$ and $T_{2}$ on the performance of the contention algorithm, we focus here on a simplified analytical cost model. This is done to derive a meaningful analysis that will drive us in the choice of these parameters and that will reveal the importance of the degree of correlation between the costs of the nodes participating in the contention. The insight gained from this simplified analysis can then be used as heuristics in more general cases. The more realistic cost model in Eq. (3) will be considered again in the performance evaluation section.

Assume to have $K \leq N$ nodes in the set $\mathcal{S}$, where $N$ is the number of devices in $\mathcal{F}$, and let us refer to $c_{j}$ as the cost associated with node $j \in \mathcal{S}$. Moreover, in order to model the cost correlation among nodes, we assume that the r.v. $C_{j}$ governing the cost of node $j\left(c_{j}\right)$ is achieved by summing two r.v.s $\bar{C}$ and $\Omega_{j}$ as follows, $C_{j}=\bar{C}+\Omega_{j}$, where $\bar{C} \in \mathcal{U}[0,1]$ and $\Omega_{j} \in \mathcal{U}[-\alpha \bar{c}, \alpha(1-\bar{c})], \alpha \in[0,1]$ and $\bar{c}$ is the actual value of the r.v. $\bar{C}$. Therefore, the cost of a generic node $j \in \mathcal{S}$ is given by a common part $\bar{C}$, which is equal for all nodes in $\mathcal{S}$, and an additive random displacement (or disturbance) $\Omega_{j} \in$ $[-\alpha \bar{c}, \alpha(1-\bar{c})]$, which is independently picked for every node in the set but that depends 


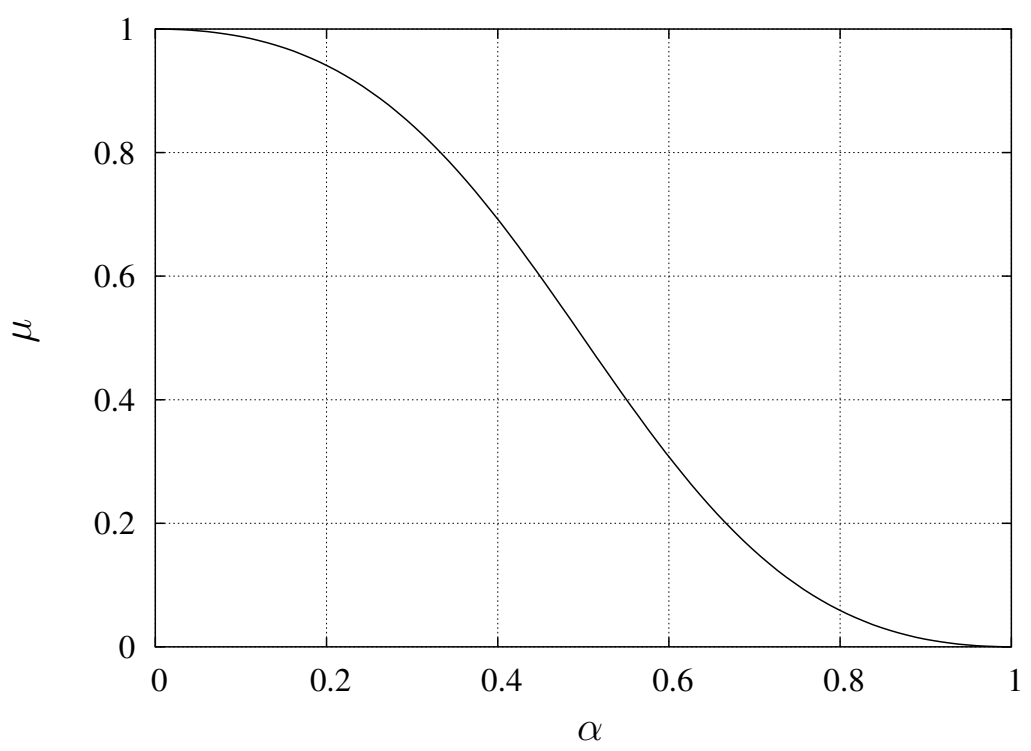

Fig. 5. Relationship between the cost correlation $\mu$ and $\alpha$.

(is conditioned) on the actual value of the r.v. $\bar{C} \cdot \bar{c}$ in our model is used to represent the common cost component of nodes in $\mathcal{S}$. Clearly, the limiting cases $\alpha=0$ and $\alpha=1$ correspond to the fully correlated case, i.e., where all nodes in $\mathcal{S}_{N}$ have the same cost $\bar{c}$, and to the independent case, i.e., where all costs are uncorrelated, respectively. This is a simple model that we introduce to mathematically derive a precise relationship between the cost correlation $\mu$ and the collision probability $P_{\text {coll }}$. We observe that the model is in general not accurate for every network condition. However, it allows to find the quantities of interest in analytical form as well as to derive useful insights on the impact of the cost correlation on the relay selection procedure. We define the correlation coefficient between any two nodes $r, s \in \mathcal{S}$ as

$$
\mu_{r, s}=\frac{\mathbb{E}\left[C_{r} C_{s}\right]-\mathbb{E}\left[C_{r}\right] \mathbb{E}\left[C_{s}\right]}{\sigma_{r} \sigma_{s}}
$$

where $\sigma_{s}^{2}=\mathbb{E}\left[\left(C_{s}-\mathbb{E}\left[C_{s}\right]\right)^{2}\right]$. By standard calculations $\mu_{r, s}$ can be derived as (see Fig. 5)

$$
\mu_{r, s}=\frac{(1-\alpha)^{2}}{(1-\alpha)^{2}+\alpha^{2}}
$$

Now, for a given device $j \in \mathcal{S}$ let us refer to $\mathcal{T}_{1}$ and $\mathcal{T}_{2}$ as the r.v.s associated with the two terms composing the back-offs $c_{j} T_{1}$ and $r_{j} T_{2}$ (see Eq. 7), respectively. Their pdfs are given by:

$$
f_{\mathcal{T}_{1}}(x)= \begin{cases}\frac{1}{\alpha T_{1}} & x \in \mathcal{I} \\ 0 & \text { elsewhere }\end{cases}
$$


where $\mathcal{I}=\left[T_{1}(1-\alpha)(1-\bar{c}), T_{1}(1-\bar{c}(1-\alpha))\right]$ and

$$
f_{\mathcal{T}_{2}}(x)= \begin{cases}\frac{1}{T_{2}} & x \in\left[0, T_{2}\right] \\ 0 & \text { elsewhere }\end{cases}
$$

Moreover, if we refer to $\mathcal{T}$ as the r.v. $\mathcal{T}_{1}+\mathcal{T}_{2}$, then we have that its pdf is given by the following Eq. (12) which is the result of the convolution of the two pdfs above:

$f_{\mathcal{T}}(x)= \begin{cases}\frac{\left[\min \left(T_{1}(1-\bar{c}(1-\alpha)), x\right)-\max \left(T_{1}(1-\alpha)(1-\bar{c}), x-T_{2}\right)\right]}{\alpha T_{1} T_{2}} & x \in \mathcal{I} \\ 0 & \text { elsewhere }\end{cases}$

At this point, we are in the position of deriving the collision probability, $P_{\text {coll }}$. In particular, for a given number $K$ of nodes in $\mathcal{S}$, for a given correlation value $\mu$ and for a given pair of parameters $\left(T_{1}, T_{2}\right), P_{\text {coll }}$ in the worst case ${ }^{8}$ is derived as

$$
P_{\text {coll }}=1-K \int_{0}^{T_{1}+T_{2}} f_{\mathcal{T}}(x)\left[1-F_{\mathcal{T}}\left(x+\tau+t_{d}\right)\right]^{K-1} \mathrm{~d} x
$$

where $F_{\mathcal{T}}(x)$ is the cdf associated with the r.v. $\mathcal{T}$, whereas $\tau$ and $t_{d}$ are the propagation delay and the time needed to detect an ongoing communication, respectively. By following the same rationale, one can easily derive the joint probability $P\{$ success \& $\min \}$ of having a successful contention, i.e., that a single node will access to the channel, and that this node is the one with the smallest cost in $\mathcal{S}$. Based on the above analytical model, in the sequel we present several important results and considerations on the impact of the cost correlation on both the collision and the success probability.

\subsection{Impact of the Cost Correlation on the Relay Election Phase}

In the following discussion, we refer to $\rho(\mathcal{S})$ as the average number of nodes in the set $\mathcal{S}$ and we average $P$ success \& $\min \}$ and $P_{\text {coll }}$ over $K$, the number of nodes in $\mathcal{S},{ }^{9}$ and $\bar{c} \in \mathcal{U}[0,1]$. As a first result, in Fig. 6 we report the metric $P$ \{success \& min $\}$ as a function of the contention parameter $T_{2}$ for $T_{2}+T_{1}=0.2$ seconds. As can be observed from the figure, the cost correlation heavily impacts on the system performance. In fact, for a given $\left(T_{1}, T_{2}\right)$ pair, $P$ \{success \& $\left.\min \right\}$ is initially decreasing as a function of $\mu$, whereas when $\mu \rightarrow 1$ it starts increasing. It is also to be stressed that the importance of selecting the minimum cost node decreases with an increasing correlation as, by definition, in such case all nodes in $\mathcal{S}$ tend to be equivalent (node costs in the limiting case $\mu=1$ become equal). In Fig. 7, we plot $P_{\text {coll }}$ as a function of $T_{2}$ for the same settings. It is interesting to note that for this metric a good choice is given by $T_{2}=0.2$ $\mathrm{s}\left(T_{1}=0\right)$. These plots reflect the impact of the balancing between the two terms in

\footnotetext{
${ }^{8}$ The worst case performance comes from the fact that in the following equation we assume to have a collision with probability one whenever more than one user sends a REPLY, i.e., we do not account for the capture and fading effects.

${ }^{9}$ This is achieved by considering the nodes in $\mathcal{S}$ to be Poisson distributed with the intensity $\rho(\mathcal{S})$.
} 


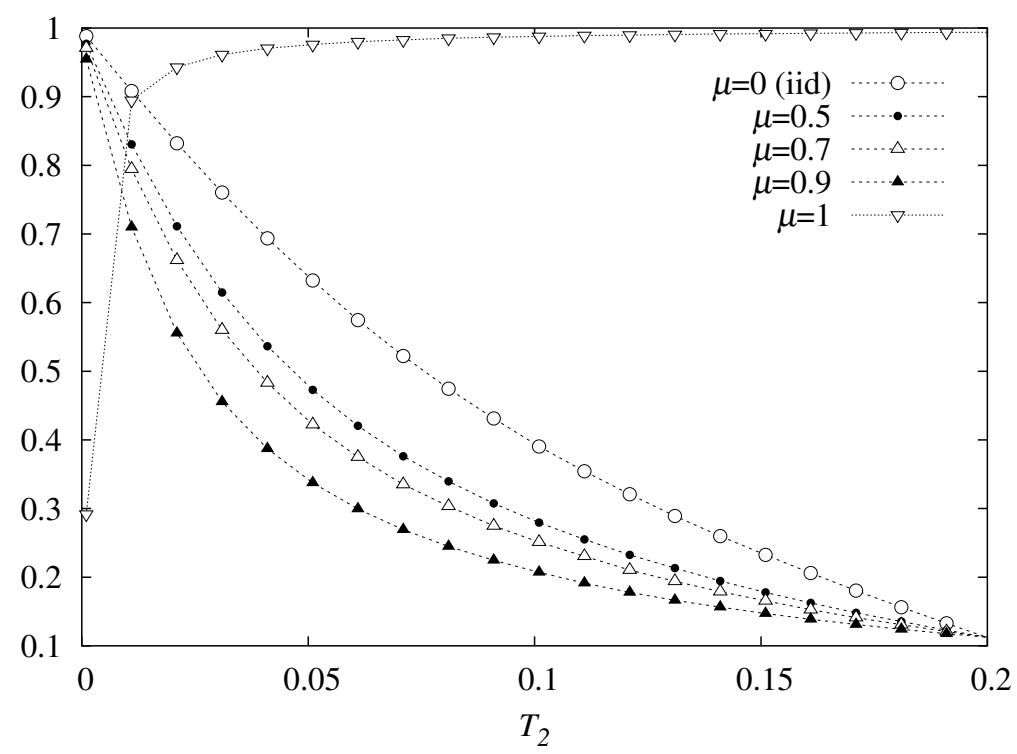

Fig. 6. $P$ \{success $\& \min \}$ as a function of $T_{2}$ by varying the cost correlation $\mu$ for $\rho(\mathcal{S})=10$ and $T_{1}+T_{2}=0.2$ seconds.

Eq (7). On the one hand, when costs are independent it is beneficial to emphasize the first term $\left(c_{j} T_{1}\right)$ so as to give priority to the lowest cost nodes. On the other hand, as costs become correlated it is worth to put more weigh on $r_{j} T_{2}$ so as to decrease the collision probability, that in this case in naturally increased due to the inherent degree of similarity among the costs (term $c_{j} T_{1}$ ). In other words, the correct balance between $c_{j} T_{1}$ and $r_{j} T_{2}$ depends on the desired trade-off between probability of picking the lowest cost node and collision probability which, in turn, depends on the underlying cost correlation structure.

The calculations in Section 5 may therefore be used to derive these metrics and select the appropriate values of $T_{1}$ and $T_{2}$ depending on our requirements (minimizing the cost associated with the relay or minimizing the collision probability). Note also that, in the most general case $T_{1}$ and $T_{2}$ might be varied between subsequent rounds of a single relay election procedure (see $T_{1}^{i}$ and $T_{2}^{i}$ in Algorithm 1). How these values can be effectively modified as a function of the round number is left for future research.

\section{Performance Evaluation}

In this section, we report some preliminary performance results by comparing our new approach with the GeRaf scheme proposed in [6]. The GeRaf framework consists of an integrated practical MAC/routing scheme based on pure advancements: its capacity of approaching the maximum possible advancements toward the destination makes GeRaf a good candidate for our investigation. Results are obtained by means of accurate Monte Carlo simulation where all packet transmissions (requests sent by the forwarder, replies sent by the candidate relay nodes as well as the final packet transmission to the relay) 


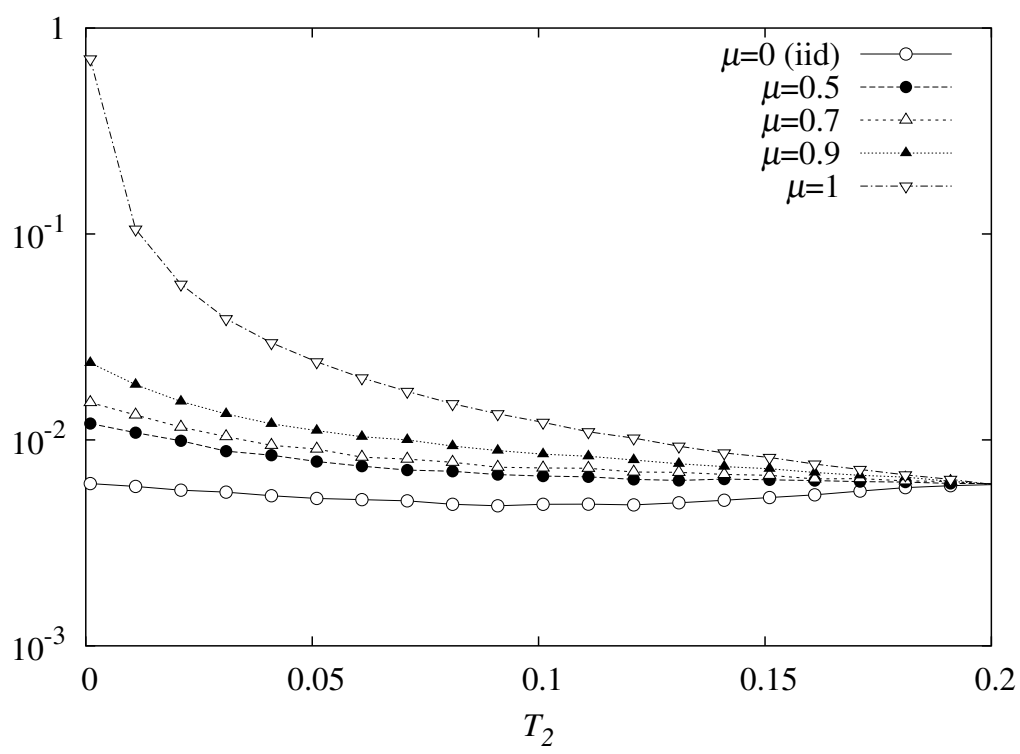

Fig. 7. $P_{\text {coll }}$ as a function of $T_{2}$ by varying the cost correlation $\mu$ for $\rho(\mathcal{S})=10$ and $T_{1}+T_{2}=$ 0.2 seconds.

are affected by fading. That is, for each packet we calculate the probability of correct reception according to Eq. (1). A study of GeRaF in the presence of fading has been presented in [13], where it was shown that the protocol is very robust to slow fading. Here, we focus on a different case, where we consider the fading channel to be completely uncorrelated between subsequent transmission/reception events. This is motivated by the following facts: 1) it represents the worst case scenario for the performance, as we can not pick a relay node with a good SNR (signal to noise ratio) metric and be sure that the good link quality will persist up to and including the actual packet transmission (forwarder $\rightsquigarrow$ relay), 2) with the latest sensor devices produced so far, due to hardware limitations as well as to the channel contention algorithms, the minimum lapse of time between subsequent transmissions/receptions is likely in the order of $200 \mathrm{~ms}, 3$ ) forward (forwarder $\rightsquigarrow$ candidate relay nodes) and backward channels (relay nodes $\rightsquigarrow$ forwarder) are likely uncorrelated due to both channel phenomena as well as hardware asymmetries [19]. Hence, the success probability associated with the transmission of a REQ by the sending entity to a node in its forwarding region likely differs from the success probability of the subsequent reply. In this case, we expect the GeRaF protocol to suffer since the successive signalling messages in a handshake are independently affected by propagation effects.

For the performance evaluation, we consider the following parameter settings: $T_{1}=$ $100 \mathrm{~ms}, T_{2}=100 \mathrm{~ms}, \xi=0.5, \bar{c} \in \mathcal{U}[0,1], \mu=0.01, n_{d}=128$ bits $^{10}, B_{r}=64 \mathrm{Kbps}$, $\rho_{n}=20$, that is, on average 20 nodes are Poisson distributed over the forwarding region $\mathcal{F}$. With these values we verified that, besides the good results that will be illustrated

\footnotetext{
${ }^{10}$ Note that the number of bits for carrier sensing depends on the hardware characteristics. Since we do not make any specific assumption here, we purposely take a conservative value.
} 


\begin{tabular}{|c|c|c|c|c|c|c|}
\hline SCHEME & $\mathbb{E}[z \mid$ suc $]$ & $\mathbb{E}\left[n_{\text {tx }} \mid\right.$ suc $]$ & $\mathbb{E}\left[n_{\text {rounds }} \mid\right.$ suc $]$ & $\mathbb{E}\left[n_{\text {cont }}\right]$ & $P_{\text {failure }}$ & $\mathbb{E}[z]$ \\
\hline GeRaf $\left(N_{r}=2\right)$ & 0.463 & 8.665 & 3.799 & 3.835 & 0.272 & 0.337 \\
GeRaf $\left(N_{r}=4\right)$ & 0.517 & 7.989 & 3.754 & 2.660 & 0.382 & 0.319 \\
GeRaf $\left(N_{r}=8\right)$ & 0.574 & 8.461 & 4.118 & 1.966 & 0.550 & 0.258 \\
& & & & & & \\
\hline New Scheme $(\delta p=0.05)$ & 0.301 & 6.806 & 3.069 & 1.827 & 0.003 & 0.300 \\
New Scheme $(\delta p=0.1)$ & 0.284 & 6.157 & 2.639 & 2.181 & 0.0005 & 0.283 \\
New Scheme $(\delta p=0.2)$ & 0.268 & 5.684 & 2.328 & 2.814 & 0.0003 & 0.267 \\
& & & & & & \\
\hline
\end{tabular}

Table 1.

in the following, our algorithm is also able to promote relay nodes with a small cost. In fact, the difference between the minimum cost among the nodes in $\mathcal{S}$ and the cost of the node elected as the relay is on average 0.08 . Further results on this issue are one of the main objectives of our future research. For the pure geographical routing scheme, we consider the version of the GeRaf protocol proposed in [6], by subdividing $\mathcal{F}$ into a given number $N_{r}$ of priority regions, according to the advancement toward the destination provided by the nodes therein. For the relay election, we consider the probabilistic contention as in [6], where the nodes in the non-empty region with the highest priority are the ones contending to act as relay. ${ }^{11}$ For what concerns the performance metrics, we consider the normalized advancement $\left(z_{\text {relay }}\right)$ provided by the relay node, the number of contention rounds ( $n_{\text {rounds }}$ ) needed to elect a relay as well as the total number of packets transmitted $\left(n_{t x}\right)$ within the entire relay election procedure, including the transmission of REQ/REPLY messages, collided packets and the final packet transmission from the forwarder to the relay node. Observe that this last metric is a good indication of the energy expenditure associated with the transmission of a single packet. Moreover, in each channel contention we account for a maximum of $N_{\max }=10$ rounds, i.e., after $N_{\max }$ failed requests (REQs) the relay election procedure is suspended and a failure is declared. $P_{\text {failure }}$ is used here to represent the failure event probability. $P_{\text {failure }}$ for the GeRaf scheme is defined similarly, i.e., as the number of packets sent by the forwarder up to the successful reply from a single node in $\mathcal{F}$ (the winner of the contention). Finally, we also track the number of devices taking part in a single contention round, $n_{\text {cont }}$. In a good channel contention algorithm, $n_{\text {cont }}$ should be limited, as much as possible, in order to keep the collision probability low.

In Table 1, we report the average values for the above performance metrics, where with $\mathbb{E}[\cdot \mid$ suc $]$ we indicate the average of the considered metric conditioned on having a successful relay election (probability $1-P_{\text {failure }}$ ), i.e., that the relay election is successfully accomplished in a number of rounds lower than or equal to $N_{\max }=10$. As can be seen from the table, the GeRaf protocol is the one showing the maximum

\footnotetext{
${ }^{11}$ Note that in our case, as the channel is faded, a region is found to be non empty by the forwarding node if its REQ is correctly decoded by at least one node in the region and if the subsequent REPLY is correctly received at the forwarder.
} 


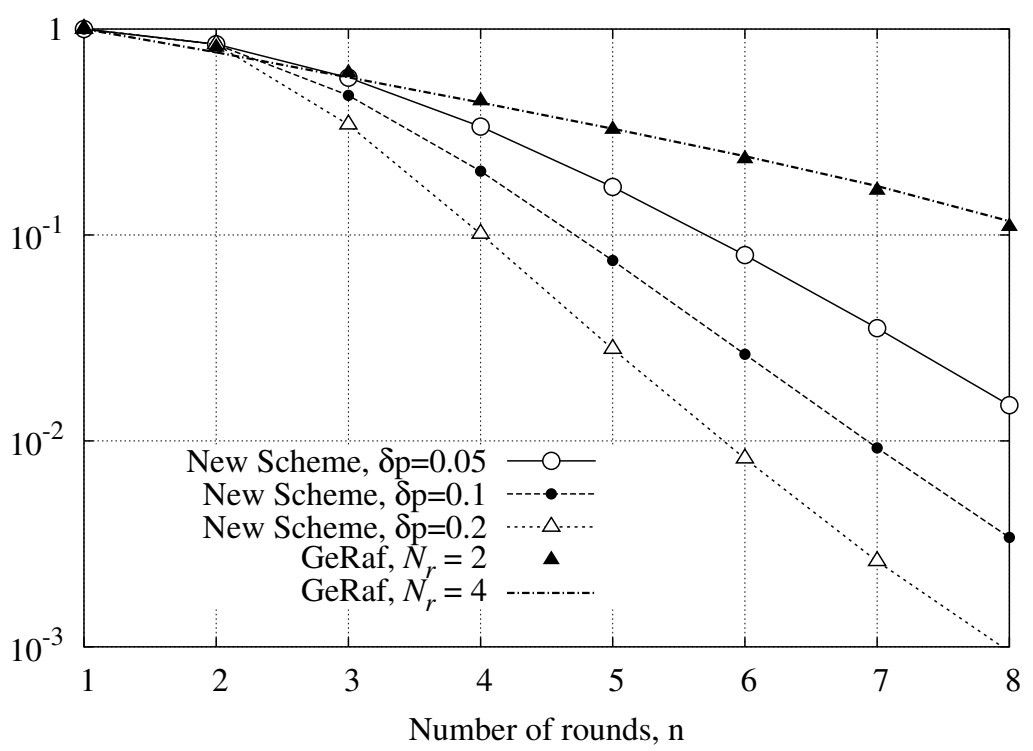

Fig. 8. Complementary cumulative distribution (ccdf) of the number of rounds $\left(n_{\text {rounds }}\right)$ needed to elect a relay node, i.e., $\operatorname{Prob}\left\{n_{\text {rounds }} \geq n\right\}$.

advancement metric $(\mathbb{E}[z \mid$ suc $])$. However, it must be observed that this metric is calculated by considering the cases where the contention is successful. In fact, the expected advancement $\mathbb{E}[z]$ is given by $\mathbb{E}[z \mid$ suc $] \times\left(1-P_{\text {failure }}\right)$. As expected, for the GeRaf protocol an increasing $N_{r}$ leads to the following consequences: 1) the average number of devices participating in the relay election $\left(n_{\text {cont }}\right)$ decreases as the size of the priority region is also decreased, 2) the failure probability increases as the forwarding node tries to elect a relay among the nodes placed close to the limit of the transmission range, 3) conditioned on a successful contention, the advancement $\mathbb{E}[z \mid$ suc $]$ also increases for the reasons illustrated in the previous point. However, as highlighted by the results shown here, when the channel is faded the maximization of the pure advancement metric has to be avoided, as the resulting success probability may become very low. In general, in the present scheme we trade pure geographical advancements for more reliability as well as a smaller number of packet transmissions (lower energy consumption) for each packet forwarding. In Fig. 8, we report the complementary cumulative distribution (ccdf) of the number of rounds needed to elect a relay node, whereas in Fig. 9 we plot the ccdf of the total number of transmissions $\left(n_{t x}\right)$ involved in a single channel contention. These statistics are conditioned on having a successful contention. As can be observed from Table 1 and Figs. 8 and 9, the available parameters (e.g., $\delta p$ ) can be varied in order to cut the desired tradeoff between advancements, reliability and energy consumption (number of transmitted packets).

We observe that the $\xi$ parameter, which govern the cost-based contention, can also be set to further improve the advancement metric, this of course will be achieved at the expense of the residual energy (see Eq. (3)). The study of the effect of this parameter as well as a the investigation of 1) multi-hop performance of the scheme and 2) impact 


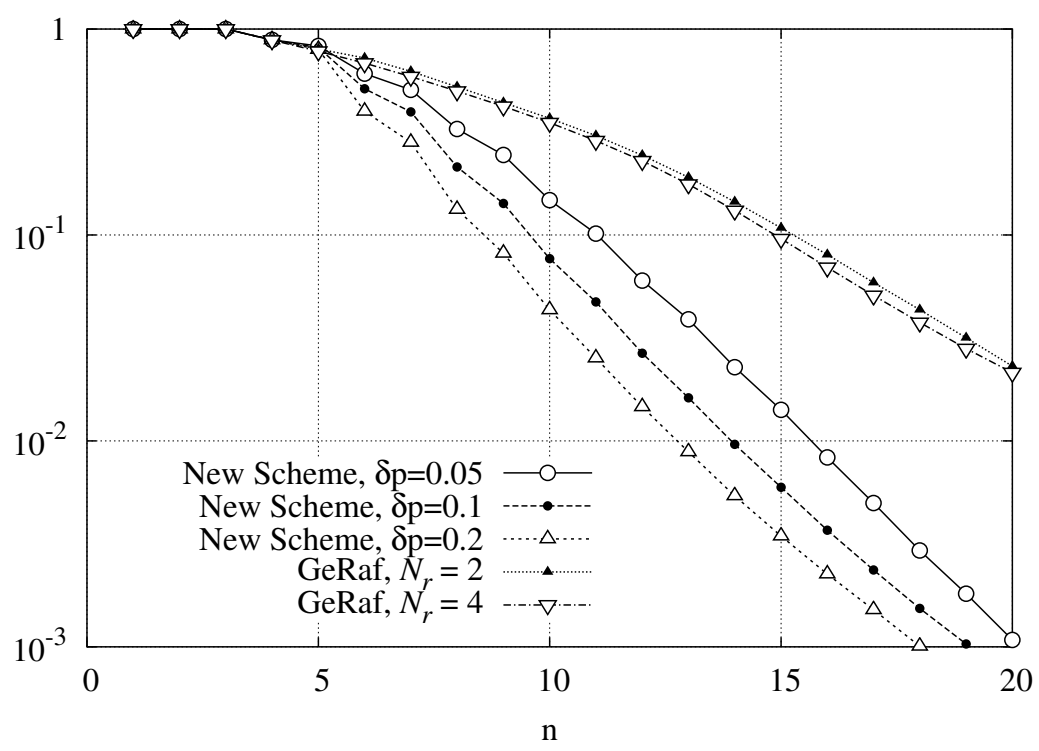

Fig. 9. Complementary cumulative distribution (ccdf) of the total number of packet transmitted $\left(n_{t x}\right)$ in a single relay election phase (including collided packets), i.e., $\operatorname{Prob}\left\{n_{t x} \geq n\right\}$.

of $\xi$ on the residual energy of the node elected as relay are the objective of future work. Finally, we can conclude that the obtained results indicate that our probabilistic filtering of the active nodes in $\mathcal{F}$ is effective in selecting the nodes with a good expected advancement metric and that the subsequent channel contention is also able to elect the relay node very quickly and considerably limiting the number of competitors accessing the channel. While here we highlighted the feasibility as well as the effectiveness of our approach, we also stress that further results on the setting of various parameters as well as a deeper investigation of the impact of the cost correlation are needed. These aspects will be addressed in our future research.

\section{Conclusions}

In this paper we discussed a novel integrated MAC/routing solution for geographical routing in wireless sensor networks. Differently from most previous contributions, we explicitly considered the fading channel statistics and we subsequently proposed a new method to deal with geographical advancements when the channel is faded. Our framework is based upon a probabilistic filtering of the awake nodes in the forwarding region. That is, based on analytically derived curves, we rule out from the contention phase the nodes that will likely lead to either unsatisfactory advancements or poor link qualities. In addition, we couple this first filtering mechanism with a novel channel contention method where back-off timers are set depending on node costs, so as to control the trade-off between the cost (e.g., residual energy) of the elected relay and the collision probability, i.e., the delay associated with the channel contention. Finally, we compare our solution with a recent scheme based on pure geographical advancements showing 
that, by taking the fading statistics into account in the relay election, good improvements can indeed be achieved. Moreover, our results confirm that pure geographical advancement toward the destination is not a good policy to be used in the presence of independent multi-path fading.

\section{References}

1. I. F. Akyildiz, W. Su, Y. Sankarasubramaniam, and E. Cayirci, "A Survey on Sensor Networks," IEEE Commun. Mag., vol. 40, no. 8, pp. 102-116, Aug. 2002.

2. M. Ilyas, The Handbook of Ad hoc Wireless Networks. CRC Press, 2002.

3. M. Mauve, J. Widmer, and H. Hartenstein, "A Survey on Position-based Routing in Mobile Ad Hoc," IEEE Network Mag., vol. 15, no. 6, pp. 30-39, Nov. 2001.

4. B. Karp and H. T. Kung, "GPSR: Greedy Perimeter Stateless Routing for Wireless Networks," in Proceedings of IEEE/ACM MobiCom 2000, Boston, MA, Aug. 2000, pp. 243 254.

5. F. Kuhn, R. Wattenhofer, Y. Zhang, and A. Zollinger, "Geometric Ad-Hoc Routing: of Theory and Practice," in Annual ACM Symposium on Principles of Distributed Computing, Zurich, Switzerland, 2003, pp. 63-72.

6. M. Zorzi and R. R. Rao, "Geographic Random Forwarding (GeRaF) for Ad Hoc and Sensor Networks: Multihop Performance," IEEE Trans. on Mobile Computing, vol. 2, pp. 337-348, Oct-Dec 2003.

7. G. Zhou, T. He, S. Krishnamurthy, and J. Stankovic, "Impact of Radio Irregularity on Wireless Sensor Networks," in ACM MobiSys 2004, Boston, Massachusetts, US, June 2004.

8. Marco Zuniga and Bhaskar Krishnamachari, "Analyzing the Transitional Region in Low Power Wireless Links," in IEEE SECON 2004, Santa Clara, CA, Oct. 2004.

9. A. Cerpa, J. L. Wong, L. Kuang, M. Potkonjak, and D. Estrin, "Statistical Models of Lossy Links in Wireless Sensor Networks," in ACM IPSN 2005, Los Angeles, CA, US, Apr. 2005.

10. B. N. Clark, C. J. Colbourn, and D. S. Johnson, "Unit Disk Graphs," Discrete Mathematics, vol. 86, no. 1-3, pp. 165-177, Aug. 1991.

11. Gordon Stüber, Principles of Wireless Communications, 2nd ed. Kluwer Academic, 1896.

12. K. Seada, M. Zuniga, A. Helmy, and B. Krishnamachari, "Energy-Efficient Forwarding Strategies for Geographic Routing in Lossy Wireless Sensor Networks," in IEEE SECON 2004, Santa Clara, CA, Oct. 2004.

13. M. Zorzi and R. R. Rao, "Energy efficient forwarding for ad hoc and sensor networks in the presence of fading," in IEEE International Conference on Communications, Paris, France, June 2004.

14. M. Zorzi and A. Armaroli, "Advancement optimization in multihop wireless networks," in IEEE VTC 2003, Orlando, Florida, US, Oct. 2003.

15. S. Lee, B. Bhattacharjee, and S. Banerjee, "Efficient Geographic Routing in Multihop Wireless Networks," in ACM MobiHoc 2005, Urbana-Champaign, Illinois, US, May 2005.

16. D. Stoyan, W. S. Kendall, and J. Mecke, Stochastic Geometry and its Applications, 2nd ed. John Wiley \& Sons, 1995.

17. R. Nelson and L. Kleinrock, "The Spatial Capacity of a Slotted ALOHA Multihop Packet Radio Network wth Capture," IEEE Trans. Commun., vol. 32, no. 6, pp. 684-694, June 1984.

18. L. Kleinrock and J. Silvester, "Optimum Transmission Radii for Packet Radio Newtorks or why Six is a Magic Number," in Proceedings of IEEE National Telecommunications Conference, Birminghan, Alabama.

19. D. Kotz, C. Newport, and C. Elliot, "The mistaken axioms of wireless-network research," Technical Report TR2003-467, July 2003, Dept. of Computer Science, Dartmouth College. 